\title{
Improved neural network 3D space obstacle avoidance algorithm for mobile robot
}

\author{
Yuchuang Tong ${ }^{1,2,3}$, Jinguo $\mathrm{Liu}^{1,2(\bowtie)}$, Yuwang Liu ${ }^{1,2}$, Zhaojie $\mathrm{Ju}^{4}$ \\ ${ }^{1}$ Stata Key Laboratory of Robotics, Shenyang Institute of Automation, \\ Chinese Academy of Sciences, Shenyang 110016, China \\ liujinguo@sia.cn \\ ${ }^{2}$ Institutes for Robotics and Intelligent Manufacturing, \\ Chinese Academy of Sciences, Shenyang 110016, China \\ ${ }^{3}$ University of the Chinese Academy of Science, Beijing 100049, China \\ ${ }^{4}$ School of Computing, University of Portsmouth, Portsmouth, PO1 3HE, UK
}

\begin{abstract}
Path planning problems are classical optimization problems in many fields, such as computers, mathematics, transportation, robots, etc., which can be described as an optimization problem in mathematics. In this paper, the mathematical model of obstacle environment is established. The characteristics of neural network algorithm, simulated annealing algorithm and adaptive variable stepsize via linear reinforcement are studied respectively. A new neural network 3D space obstacle avoidance algorithm for mobile robot is proposed, which solves the problem of the computational duration and minimum distance of the traditional neural network obstacle avoidance algorithm in solving the optimal path. According to the characteristics of the improved neural network algorithm, it is fused with a variety of algorithms to obtain the optimal path algorithm that achieves the shortest path distance and meets the requirements of obstacle avoidance security. The simulation experiment of the algorithm is simulated by Matlab. The results show that the improved neural network spatial obstacle avoidance algorithm based on the multiple algorithms proposed in this paper can effectively accelerate the convergence speed of path planning, realize the minimum path distance, and achieve very good path planning effect.
\end{abstract}

Keywords: Global path planning, Obstacle avoidance algorithm, Improved neural network algorithm, Adaptive variable stepsize, Simulated annealing

\section{Introduction}

Path planning has a wide range of applications in many areas, such as traffic, logistics, routing search for communication systems, robot walking route planning, etc. $[1,2]$. Demand for drones and underwater vehicles is growing, the $3 \mathrm{D}$ path planning issues involved are complex, so the use at civil and commercial areas is still very limited $[3,4]$. The path planning problem can be expressed as a mathematical multiobjective optimization problem, so the path planning problem is transformed into solving the corresponding mathematical optimization problem $[5,6]$. At present, in the 
study of global path planning problems based on environmental prior information, the typical methods proposed in $[7,8,9]$ are visibility graph, artificial potential field method and so on. The advantage of the visibility graph is that the shortest path can be obtained, but there is a drawback of the combined explosion problem. The artificial potential field method overcomes the combinatorial explosion problem by finding the minimum value point of the path point energy function, avoiding the combined explosion problem, but has the problem of local minimum value and is not suitable for finding the shortest path. Since the computational time of deterministic algorithms and complexity increases exponentially with the dimensions of the configuration space, these algorithms cannot provide a reliable solution for real-time applications $[10,11]$.

In order to solve the problem of mobile robot movement in $3 \mathrm{D}$ environment, it is an effective method to apply neural network to mobile robot motion trajectory automatic generation and mobile robot path planning in literature. Neural network path planning algorithm introduces the network structure method according to the basic idea of the artificial potential field method. The calculation is simple and can avoid some local extreme values $[12,13]$. In this paper, the simulated annealing algorithm is used to optimize the collision penalty function of the neural network algorithm. The adaptive variable step size algorithm is used to optimize the iterative step size parameters of the neural network algorithm. The two algorithms complement each other to further achieve the optimization effect. Therefore, based on the algorithm, an improved algorithm for neural network path planning based on adaptive variable stepsize via linear reinforcement and simulated annealing algorithm is proposed. It not only has the advantages of the original algorithm, but also runs faster than the original algorithm. In particular, the planned collision-free path can quickly reach the shortest path of the destination, basically meeting the needs of real-time path planning.

The rest of this paper is structured as follows: Sect. 2 is the main part of this paper. It will briefly introduce the principles and specific steps of traditional neural network obstacle avoidance algorithms, and show the description of obstacles and ob-stacle modeling. Sect. 3 improved neural network 3D obstacle avoidance path planning algorithm is proposed, which solves the problem of the computational duration and minimum distance when the traditional algorithm solving the optimal path. And in Sect. 4 the problem of the improved neural network in solving the nonconvex optimization problem is obtained, and the algorithm is combined with the continuous simulated annealing algorithm. Sect. 5 based on the previous algorithm, the adaptive selection of the step size is realized by the linear reexcitation learning method. Its future development trends are studied in Sect. 6.

\section{Related work}

\subsection{Principle of neural network obstacle avoidance algorithm}

As a highly parallel distributed system, neural network provides a great possibility to solve the problem of high real-time requirements of robot systems, and is applied to intelligent autonomous mobile robot navigation and path planning. In this section, the 
idea of solving the path planning problem is using neural network to describe the environmental constraints and calculate the collision energy function. The sum of the collision energy function and the distance function of the iterative path point set is used as the optimization objective function, and the optimization objective function is obtained. The extremum determines the equation of motion for the point set and eventually makes the set of iterative path points tend to the optimal planning path.

\subsection{Neural network modeling of obstacles}

The collision penalty function of a path is defined as the sum of the collision penalty functions of each path point, and the collision penalty function of one point is obtained through its neural network representation of each obstacle. The obstacle is assumed to be a polygon, which can be represented by a set of linear inequalities, so that the points in the obstacle must satisfy the limits of all inequalities. Fig. 1(a) shows neural network from a penalty function to an obstacle. The bottom two nodes represent the coordinates $\mathrm{x}$ and $\mathrm{y}$ of the given path point respectively. Each node of the middle layer corresponds to an inequality constraint of the obstacle. The connection weight coefficients of the bottom layer and the middle layer are equal to the $x$ and $y$ front of the inequality. The coefficient of each node in the middle layer is equal to the constant term in the corresponding inequality. The connection weight from the middle layer to the top layer is 1 , and the threshold of the top node is taken as the number of inequalities minus the negative number after 0.5 .

The operational relationships in the neural network model are:

$$
\begin{gathered}
C=f\left(I_{0}\right) \\
I_{0}=\sum_{m=1}^{M} O_{H m}+\theta_{T} \\
O_{H m}=f\left(I_{H m}\right) \\
I_{H m}=w_{x m}+x_{i}+w_{y m} y_{i}+w_{z m} z_{i}+q_{H m}
\end{gathered}
$$

Where $x_{i}, y_{i}$ are the horizontal and vertical coordinates of the path point, $\mathrm{C}$ is the top node output, $I_{0}$ is the top node input, $\theta_{T}$ is the top node threshold, $O_{H m}$ is the output of the m-th node middle layer, $I_{H m}$ is the m-th node the middle layer input, $\theta_{H m}$ is the threshold of the m-th node of the middle layer, $w_{x m}, w_{y m}$ is the coefficient of the $\mathrm{m}$-th inequality constraint. The sigmoid function is selected as the excitation function shown in equation (5).

$$
f(x)=\frac{1}{1+e^{-x / T}}
$$

Fig. 1(c) shows the shape of the single obstacle collision penalty function in the two-dimensional obstacle environment of the mobile robot obtained by the simulation 
of the collision penalty function, which corresponds to the environment shown in Fig. 1(b).

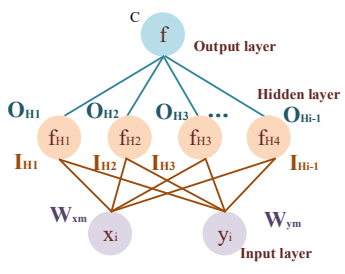

(a)

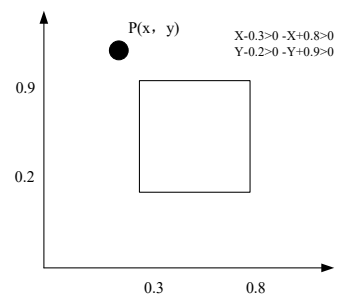

(b)

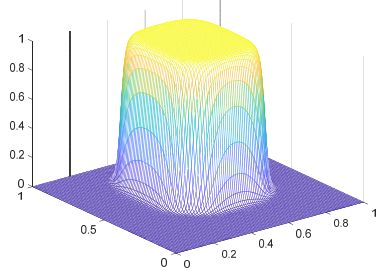

(c)

Fig. 1. (a) Neural network model of a single obstacle. (b) Single obstacle model. (c) Single obstacle collision penalty function shape in two-dimensional obstacle environment.

The working environment of mobile robots often has multiple obstacles, and one path is also composed of multiple path points, so the collision penalty function of one path point is defined as the sum of the collision penalty functions of all intermediate path points on the path. The collision penalty function of a point is obtained through its neural network representation of each obstacle. Fig. 2(a) shows a neural network model of multiple obstacle environments. In this way, as long as the x coordinate and the $y$ coordinate of each intermediate point of the path are sequentially input to each layer, the model output is the total energy of the collision penalty function corresponding to the entire path to be adjusted.

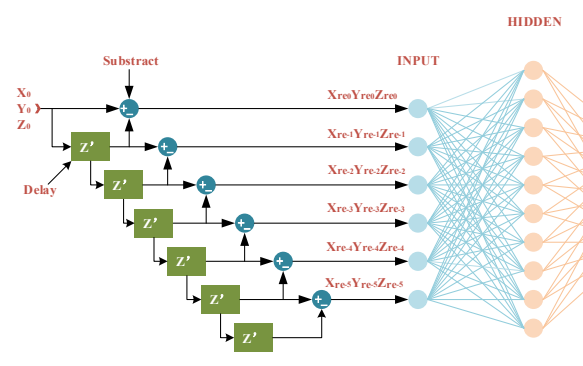

(a)

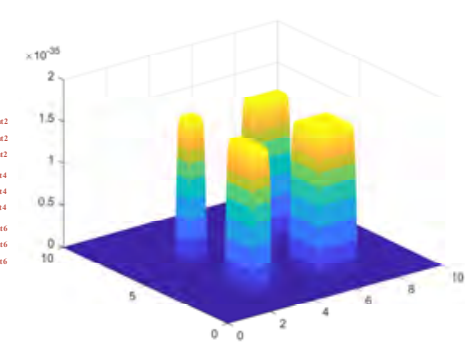

(b)

Fig. 2. (a) Multiple obstacle model. (b) Multi-obstacle collision penalty function shape in 2D obstacle environment.

Based on the simulation results of Fig. 2(b) and Fig. 1(c), it can be concluded that the collision energy function of the iterative path point set can accurately describe the obstacle environment of the mobile robot. Combined with the distance function of another factor that path planning should consider, the obstacle environments can successfully realize the static environment path planning task of mobile robot.

\subsection{Path point motion equation}

The entire path corresponds to the energy of the collision function as follow, 


$$
E_{c}=\sum_{i=1}^{N} \sum_{k=1}^{K} C_{i}^{k}
$$

Where $K$ is the number of obstacles, $N$ is the number of path points, $C_{i}^{k}$ represents the collision function of the $\mathrm{i}$-th path point $\mathrm{P}\left(x_{i}, y_{i}, z_{i}\right)$ against the $k$-th obstacle.

The energy corresponding to the length of the path is defined as the sum of the squares of the lengths of all the line segments, for all path points $\mathrm{P}\left(x_{i}, y_{i}, z_{i}\right)$, that is

$$
E_{l}=\sum_{i=1}^{N-1}\left|\left(x_{i+1}-x_{i}\right)^{2}+\left(y_{i+1}-y_{i}\right)^{2}+\left(z_{i+1}-z_{i}\right)^{2}\right|
$$

The total energy function of the entire path is defined as

$$
E=w_{l} E_{l}+w_{c} E_{c}
$$

Where $w_{l}$ and $w_{c}$ respectively represent the weighting of each part.

The above analysis shows that the shorter the path, the smaller the energy function $E$ and the smaller the collision penalty function value, and the farther the path is from the center of the obstacle, the smaller the energy function $E$ is.

If $E$ is used to derive the derivative of time

$$
\begin{aligned}
\dot{E} & =\sum_{i}\left(\nabla_{p i} E\right)^{T} p_{i}^{\prime} \\
& =\sum_{i}\left\{\left[w_{l}\left(\frac{\partial L_{i}^{2}}{\partial x_{i}}+\frac{\partial L_{i-1}^{2}}{\partial x_{i}}\right)\right]+w_{c} \sum_{k} \frac{\partial C_{i}^{k}}{\partial x_{i}}\right\} \dot{x}_{i}+\left[w_{l}\left(\frac{\partial L_{i}^{2}}{\partial y_{i}}+\frac{\partial L_{i-1}^{2}}{\partial y_{i}}\right)\right] \\
& \left.\left.\left.+w_{c} \sum_{k} \frac{\partial C_{i}^{k}}{\partial y_{i}}\right\} \dot{y}_{i}+\left[w_{l}\left(\frac{\partial L_{i}^{2}}{\partial z_{i}}+\frac{\partial L_{i-1}^{2}}{\partial z_{i}}\right)\right]+w_{c} \sum_{k} \frac{\partial C_{i}^{k}}{\partial z_{i}}\right\} \dot{z}_{i}\right\}
\end{aligned}
$$

And the dynamic equation of motion about the point $\mathrm{P}\left(x_{i}, y_{i}, z_{i}\right)$ is

$$
\begin{aligned}
& \dot{x}_{i}=-\eta_{1}\left\{2 w_{l}\left(2 x_{i}-x_{i-1}-x_{i+1}\right)+w_{c} \sum_{k=1}^{K} f^{\prime}\left[\left(T_{I}\right)_{i}^{k}\right]\left[\sum_{m=1}^{M} f^{\prime}\left[\left(I_{H m}\right)_{i}^{k}\right] w_{x m}^{k}\right]\right\} \\
& \dot{y}_{i}=-\eta_{1}\left\{2 w_{l}\left(2 y_{i}-y_{i-1}-y_{i+1}\right)+w_{c} \sum_{k=1}^{K} f^{\prime}\left[\left(T_{I}\right)_{i}^{k}\right]\left[\sum_{m=1}^{M} f^{\prime}\left[\left(I_{H m}\right)_{i}^{k}\right] w_{y m}^{k}\right]\right\} \\
& \dot{z_{i}}=-\eta_{1}\left\{2 w_{l}\left(2 z_{i}-z_{i-1}-z_{i+1}\right)+w_{c} \sum_{k=1}^{K} f^{\prime}\left[\left(T_{I}\right)_{i}^{k}\right]\left[\sum_{m=1}^{M} f^{\prime}\left[\left(I_{H m}\right)_{i}^{k}\right] w_{z m}^{k}\right]\right\}
\end{aligned}
$$

Where

$$
f^{\prime}(g)=\frac{1}{T}(g)(1-f(g))
$$


And

$$
\begin{aligned}
& \dot{x}_{i}=-\eta_{2}\left(2 x_{i}-x_{i-1}-x_{i+1}\right) \\
& \dot{y}_{i}=-\eta_{2}\left(2 y_{i}-y_{i-1}-y_{i+1}\right) \\
& \dot{z}_{i}=-\eta_{2}\left(2 z_{i}-z_{i-1}-z_{i+1}\right)
\end{aligned}
$$

\subsection{Traditional neural network obstacle avoidance algorithm steps}

The initial path point sequence selects a uniformly distributed point sequence on the line connected from the start point to the end point. The entire energy is a function of the various path points, moving each path point in a direction that reduces energy. In the traditional path planning algorithm, different dynamic equations of motion are selected according to different locations of the path points located inside and outside the obstacle.

First, three assumptions are made here: (1) The obstacle is a plane figure enclosed by polygons. (2) The robot is a circular point robot, and the size of the obstacle has been appropriately expanded according to the radius of the robot. (3) The obstacle is static.

The traditional neural network obstacle avoidance algorithm steps are as follows:

Step 1. Enter the coordinates of the starting point $\mathrm{P}\left(x_{1}, y_{1}, z_{1}\right)$ and the target point $\left(x_{N}, y_{N}, z_{N}\right)$. For $t=0$, the initial path is generally taken as a point array uniformly distributed on the straight line from the starting point to the target point.

$$
\begin{aligned}
& x_{i}=x_{1}+i\left(x_{N}-x_{1}\right) /(N-1) \\
& y_{i}=y_{1}+i\left(y_{N}-y_{1}\right) /(N-1) \\
& z_{i}=z_{1}+i\left(z_{N}-z_{1}\right) /(N-1)
\end{aligned}
$$

Step 2. For the path point $\mathrm{P}\left(x_{i}, y_{i}, z_{i}\right), \mathrm{i}=2,3, \ldots, \mathrm{N}-1$, the parameters are $w_{l}=w_{c}=0.5, \eta_{1}=0.1, \eta_{2}=2.5$,

If $\mathrm{P}\left(x_{i}, y_{i}, z_{i}\right)$ is in the obstacle, move according to equation (10) (12),

If $\mathrm{P}\left(x_{i}, y_{i}, z_{i}\right)$ is outside the obstacle, move according to equation (14) (16).

Step 3. Repeat step 2 until the path converges.

\section{Improved neural network obstacle avoidance algorithm}

\subsection{Principle of improved neural network obstacle avoidance algorithm}

In each iteration of the improved algorithm, if the line connecting any one of the path points and the target point does not collide with the obstacle, the coordinates of all the 
path points following the path point take the same value as the coordinates of the path point. This method allows the path to converge quickly to the shortest path without collision. The improved algorithm avoids the modeling of space by collision detection of sampling points in the state space, and can effectively solve the path planning problem of high dimensional space and complex constraints.

\subsection{Steps of improved neural network obstacle avoidance algorithm}

The improved fast neural network path planning algorithm is given below:

Step1. After determining the starting point and the target point, connect the starting point and the target point and evenly take $n$ intermediate path points, same as the traditional algorithm.

Step2. Connect the target point to the first, second, and $n$-th path points, and determine whether the two points are in collision with the obstacle.

Step3. If the connecting line of the two points does not collide with the obstacle, then all the path point coordinates after the path point are taken to be the same as the path point.

Step4. If the two-point connection line collides with the obstacle, return to step 1, recalculate the target point according to the path planning method of the neural network, and connect with the target point again until the target point and the intermediate path point connection does not collide with the obstacle. Here, in order to solve the safety problem of the path planning of the mobile robot, the obstacle can be subjected to a series of puffing treatment.

The execution result of the path planning algorithm is shown in Fig. 3.

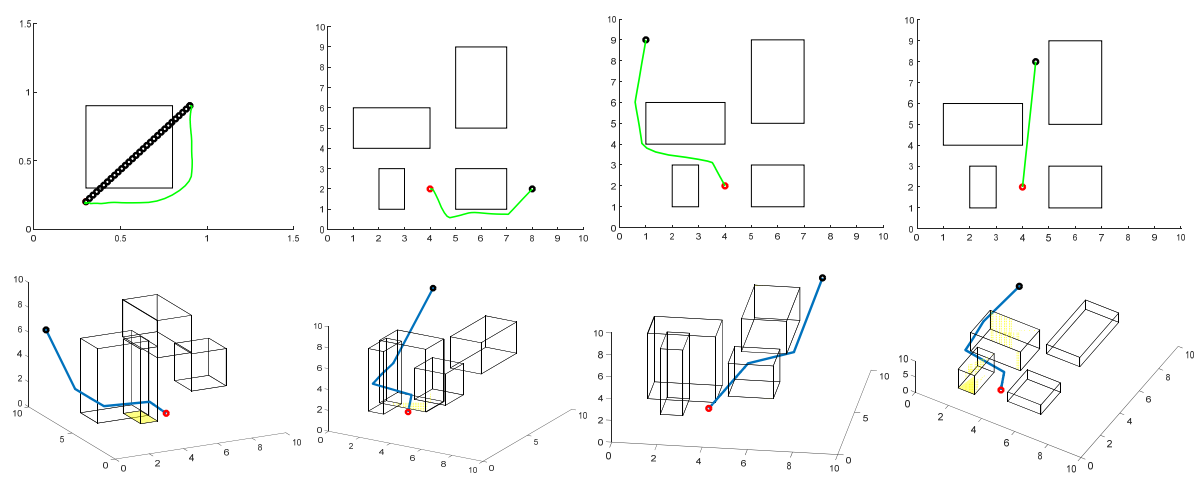

Fig. 3. Above: Single obstacle and multi-obstacle obstacle avoidance planning in twodimensional space. Below: Multi-obstacle obstacle avoidance in three-dimensional space.

\subsection{Comparison of algorithm performance}

The following Fig. 4 shows the trajectory comparison of the traditional neural network obstacle avoidance algorithm and the improved neural network obstacle avoidance algorithm. The red line is the traditional algorithm and the green line is the improved algorithm. 


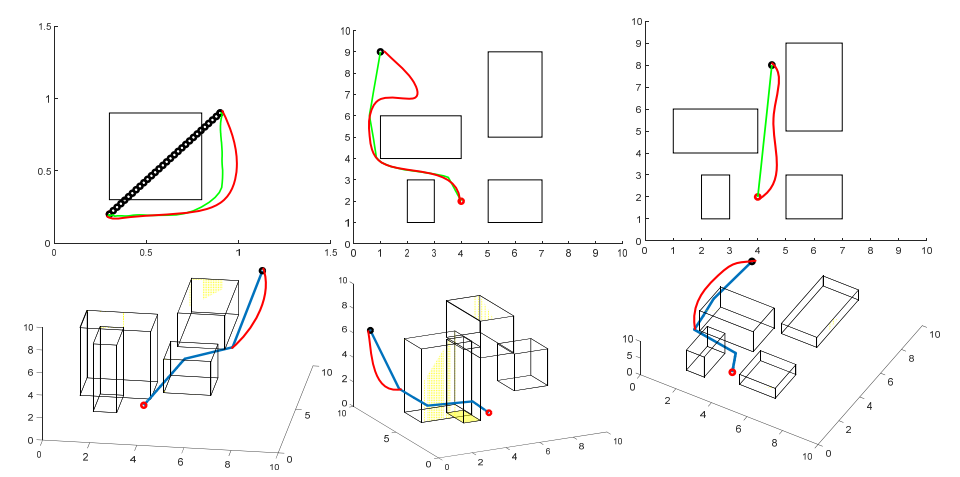

Fig. 4. Obstacle avoidance path planning algorithm based on traditional neural network method and improved neural network obstacle avoidance algorithm

Since the traditional neural network will judge whether each path point is outside the obstacle in each iteration step, the improved algorithm only needs to judge whether the connection line between each path point and the target point collides with the obstacle. If the line do not collide, only other path points need to be judged to be related to obstacles, which saves running time and iteration times. By comparison, the path planned by the traditional neural network obstacle avoidance algorithm is not the optimal path, the path is long, the number of iterations is large, and the running time is long. The improved neural network obstacle avoidance algorithm can ensure the path is smooth and shortest, and can also reduce unnecessary iterative and obstacle collision detection steps, save path planning time.

\section{Simulated annealing algorithm}

\subsection{Principle of simulated annealing algorithm}

Simulated annealing is a random search method that is inspired by the annealing process. The basic idea is to first set the temperature to a level high enough that most of the random motion directions are feasible, and a relatively low target area can be found in a relatively large space. As the temperature slowly decreases according to certain rules, the probability that each direction is selected will become different, and of course the accuracy of the search will continue to increase.

\subsection{Parameter setting}

When using the simulated annealing algorithm to solve the optimization problem, whether the parameter setting is reasonable or not greatly affects the performance of the algorithm. However, in practical applications, the algorithm is only required to give a solution that satisfies the accuracy in a reasonable time. In order to meet the requirements of accuracy and efficiency, it is necessary to analyze and design several main parameters of the simulated annealing algorithm and set the size reasonably.

- Initial temperature $T_{0}$ selection. 
When the value of the parameter $\mathrm{T}$ is different, the model diagram of the obstacle is as shown in Fig. 5. It can be obtained through simulation that when the parameter $\mathrm{T}$ taken in (5) is appropriate, the collision penalty function can accurately reflect the distribution of obstacles in the environment where the mobile robot is located.

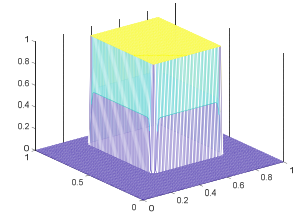

(a)

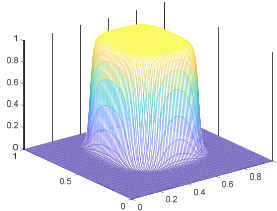

(b)

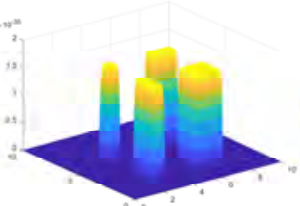

(c)

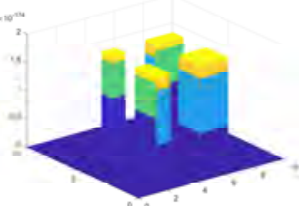

(d)

Fig. 5. (a) $3 \mathrm{D}$ model of single obstacle at $\mathrm{T}=0.01$. (b) $3 \mathrm{D}$ model of single obstacle at $\mathrm{T}=0.05$. (c) 3D model of multiple obstacles at $\mathrm{T}=0.01$. (d) $3 \mathrm{D}$ model of multiple obstacles at $\mathrm{T}=0.05$.

\section{- Selection of temperature drop function.}

Using the relationship between the parameter $\mathrm{T}$ and the penalty function energy surface, by starting with a higher "temperature" $\mathrm{T}$, the path point is coarsely adjusted, then gradually decreasing $\mathrm{T}$, and fine-tuning the path point to achieve simulated annealing effect.

It can be shown that when the "temperature" $\mathrm{T}$ changes according to the following rules:

$$
\frac{T_{a}(t)}{T_{0}}=\frac{1}{\log (1+t)}
$$

Since the convergence speed is slower according to the above formula, the following simulated annealing law can be adopted:

$$
T(t)=\frac{T_{a}}{1+t}
$$

Adopting this rule greatly accelerates the convergence speed and shortens the calculation time of path planning.

\subsection{Steps of simulated annealing and improved algorithm combined}

In this algorithm, the simulated annealing algorithm is combined with the improved neural network obstacle avoidance algorithm obtained in Chapter 3. The main steps can be described as follow:

Step1. According to the method in Sect. 3 to generate uniform track points. And determine the initial temperature $T_{0}$.

Step2. For the path points $\mathrm{P}\left(x_{i}, y_{i}, z_{i}\right)$, the derivatives of the points in the $\mathrm{x}, \mathrm{y}, \mathrm{z}$ direction are calculated according to the improve algorithm, thereby performing iterative operations and moving . 
Step3. Repeat step 2 until the temperature is reached at the internal circulation stop condition (sampling stability condition), then go to step 4;

Step4. Decrease the temperature $T$ according to equation (21) and proceed to step 2. The search is terminated when the temperature $T$ drops enough to converge the entire path to the global minimum.

\subsection{Comparison of algorithm performance}

In this paper, the simulation platform is used as the Matlab program, and the comparison of the results is shown in Table 1. In the table, the improved algorithm refers to the improved neural network algorithm in Chapter 3, and the optimization algorithm is combination of the simulated annealing method and the improved algorithm in Chapter 3.

Table 1. Comparison of results of different algorithms

\begin{tabular}{lll}
\hline Algorithm & Number of iterations & Time consuming /s \\
\hline Improved algorithm & 310 & 1.319 \\
Optimized algorithm & 140 & 0.688 \\
\hline
\end{tabular}

In the case where the results are same, the latter is nearly $1 / 2$ faster than the former. The results in the above table show that the neural network is combined with the continuous simulated annealing algorithm to solve the non-convex optimization problem by using the local optimization ability. The results show that the combination of neural network and annealing algorithm overcomes the limitation of neural network for solving nonconvex optimization problems, and it is more efficient.

\section{$5 \quad$ Adaptive variable step size via linear reinforcement}

\subsection{Principle of adaptive variable step size via linear reinforcement}

In the improved NA algorithm in Sect. 3, the step rolling $\eta_{1}, \eta_{2}$ is constant. When the selection of $\eta_{1}, \eta_{2}$ is too large, the path planning will be divergent. When the selection of $\eta_{1}, \eta_{2}$ is too small, the convergence speed is slow, especially for different planning path, there is no universal fixed step size, and sometimes it has to be reselected.

In this paper, we combine this algorithm with the improved algorithm obtained in the previous chapter, use the idea of re-energized learning to find an adaptive variable step size algorithm to realize the automatic selection of step size, accelerate the convergence speed of NA algorithm, and reduce the number of iterations of path planning. The basic idea of the adaptive variable step size algorithm is: if two iterations are repeated, the gradient direction foot is opposite to the sign, which means "falling too much", the step size is too large, the step size should be reduced, conversely, if it is consecutive iteration, the sign of the gradient direction is the same, which means that the decline is slow and has not reached a minimum, the step size should be in- 
creased. This paper adopts the following method in the combined algorithm, as the equation $(22) \sim(23)$.

$$
\begin{aligned}
& \eta_{1}(n+1)=\eta_{1}(n)+\lambda \times \operatorname{sgn}((E(n)-E(n-1)) \times(E(n-1)-E(n-2))) \\
& \eta_{2}(n+1)=\eta_{2}(n)+\lambda \times \operatorname{sgn}((E(n)-E(n-1)) \times(E(n-1)-E(n-2)))
\end{aligned}
$$

Where $\lambda$ is a constant ranging from 0.001 to 0.003 . The above algorithm actually utilizes and memorizes the symbol change information in the gradient direction.

\subsection{Comparison of algorithm performance}

The comparison of the results is shown in Table 2. In the table, the optimization algorithm is combination of the adaptive variable step size algorithm and the chapter 4 optimization algorithm.

Table 2. Comparison of results of different algorithms

\begin{tabular}{lll}
\hline Algorithm & Number of iterations & Time consuming /s \\
\hline Improved algorithm & 310 & 1.319 \\
Optimized algorithm & 30 & 0.0109 \\
\hline
\end{tabular}

Using this combined algorithm for path planning, the same shortest obstacle avoidance path as in Fig. 3 is obtained, and the convergence speed of path planning is nearly 10 times faster. The results in the above Table 2 show that the adaptive selection of the step size is realized by the linear re-excitation learning method, the path planning of the adaptive variable-step neural network path planning algorithm using adaptive variable stepsize via linear reinforcement algorithm is faster than the improved NA algorithm in the previous sections.

\section{Conclusion}

This paper mainly introduces an improved neural network obstacle avoidance method that combines multiple algorithm. which saves the planning time, effectively speeds up the convergence of path planning, and achieves the minimum path distance, achieving very good path planning results. The innovations of this paper includes: (a) A new neural network 3D space obstacle avoidance algorithm for mobile robot is proposed, which solves the problem of the computational duration and minimum distance of the traditional neural network obstacle avoidance algorithm in solving the optimal path. (b) The characteristic of the algorithm is combined with a variety of algorithms to obtain an optimal path algorithm that achieves the shortest path distance and meets the requirements of obstacle avoidance security. The improved neural network spatial obstacle avoidance algorithm based on the proposed algorithm can combine the advantages of each algorithm to achieve the path obstacle avoidance planning. (c) The characteristics of neural network algorithm, simulated annealing algo- 
rithm and linear re-energized adaptive variable step size algorithm are studied respectively, and their advantages are combined to form a new algorithm.

The future work mainly includes: (a) Adding fuzzy control algorithm to neural network path planning algorithm to solve obstacle avoidance problems in dynamic environments. (b) Combining powerful visual directions to solve the problem of obstacle avoidance in unknown 3D space.

\section{References}

1. Foux, G. , Heymann, M., Bruckstein, A.: Two-dimensional robot navigation among unknown stationary polygonal obstacles. IEEE Transactions on Robotics and Automation 9(1), 96-102 (1993).

2. Luo, Y. F.,Liu, J. G., Gao, Y., Lu, Z. L.: Smartphone-controlled robot snake for urban search and rescue. In: The 7th International Conference on Intelligent Robotics and Application (ICIRA), pp. 352-363.(2014).

3. Khosla, P., Volpe R.: Superquadric artificial potentials for obstacle avoidance and approach. In: IEEE International Conference on Robotics \& Automation, pp. 1778-1784. IEEE (2002).

4. Lee, M., Park, M.: Artificial potential field based path planning for mobile robots using a virtual obstacle concept. In: IEEE/ASME International Conference on Advanced Intelligent Mechatronics, pp. 735-740. IEEE (2003).

5. Zhang, X. ,Liu, J. G.: Effective motion planning strategy for space robot capturing targets under consideration of the berth position. Acta Astronautica 148, 403-416 (2018).

6. Fei, K., YaoNan, W.: Robot Path Planning Based on Hybrid Artificial Potential Field/Genetic Algorithm. Journal of System Simulation 18 (3), 774-777 (2006).

7. Yun, S. C., Ganapathy, V., Chong, L. O.: Improved genetic algorithms based optimum path planning for mobile robot. In: International Conference on Control Automation Robotics \& Vision,pp. 1565-1570. IEEE (2011) .

8. Wzorek, M., Doherty, P.: Reconfigurable Path Planning for an Autonomous Unmanned Areal Vehicle. In: Int. Conf. Hybrid Information Technology (ICHIT'06), pp. 438-441. IEEE (2006).

9. Liu, T. L., Wu, C.D., Li, B., Liu, J.G.: The Adaptive Path Planning Research for a ShapeShifting Robot Using Particle Swarm Optimization. In: 5th International Conference on Natural Computation, pp.324-328. IEEE (2009).

10. Ge, S. S., Cu, Y. J.: New Potential Functions for Mobile Robot Path Planning. IEEE Transactions Robot Automat 16 (5), 615-620 (2000).

11. Yang, S., Meng, M.: Real-time Collision-free Path Planning of Robot Manipulators using Neural Network Approaches. Autonomous Robots 9 (1), 27-39 (2000).

12. Xu, X., Xie, J., Xie, K.: Path Planning and Obstacle-Avoidance for Soccer Robot Based on Artificial Potential Field and Genetic Algorithm. In: World Congress on Intelligent Control \& Automation, pp. 3494-3498. IEEE (2006).

13. Li, Q., Zhang, W., Yin, Y., Wang, Z., Liu, G.: An Improved Genetic Algorithm of Optimum Path Planning for Mobile Robots. In: 6th International Conference on Intelligent Systems Design and Applications, pp. 637-642. IEEE (2006). 